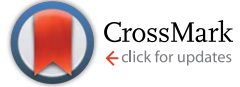

Cite this: RSC Adv., 2017, 7, 14099
Received 5th January 2017 Accepted 22nd February 2017

DOI: $10.1039 / \mathrm{c} 7 \mathrm{ra00154a}$

rsc.li/rsc-advances

\section{SERS-based immunoassay on 2D-arrays of Au@Ag core-shell nanoparticles: influence of the sizes of the SERS probe and sandwich immunocomplex on the sensitivity $\uparrow$}

\author{
Kullavadee Karn-orachai, ${ }^{\text {ab }}$ Kenji Sakamoto, ${ }^{a}$ Rawiwan Laocharoensuk, ${ }^{c}$ \\ Suwussa Bamrungsap, ${ }^{\mathrm{C}}$ Tararaj Dharakul ${ }^{\mathrm{cd}}$ and Kazushi Miki ${ }^{\star a b}$
}

We report a sandwich-type SERS-based immunoassay using a two-dimensional (2D) array of gold core@silver shell (Au@Ag) nanoparticles (NPs) as the SERS substrate and antibody-conjugated gold NPs labeled with 4mercaptobenzoic acid (MBA) as the SERS probes. To achieve highly sensitive detection, the size of the SERS probes was first optimized for the immunoassay of Human-lgG $(\mathrm{H}-\lg G)$, where the Au core size of the SERS probes was varied from 26 to $110 \mathrm{~nm}$ in diameter. The maximum SERS intensity was observed at an Au core size of $53 \mathrm{~nm}$. Then, the influence of the size of the sandwich immunocomplexes on the sensitivity was examined by performing sandwich SERS immunoassays for $\mathrm{H}$-lgG and prostate-specific antigen (PSA) using SERS probes with $53 \mathrm{~nm}$ Au core size. The sensitivity improvement by using the SERS substrate (2D-array of AuaAg NPs) instead of an Au evaporated film, which was used as a reference substrate, was evaluated for each immunoassay. The sensitivity improvement for $\mathrm{H}$-lgG and PSA detection was 2.3-fold and 6.4-fold, respectively. The larger sensitivity improvement for the PSA system can be attributed to the smaller immunocomplex of PSA; the shorter separation distance between the SERS probes and the SERS substrate induces stronger plasmon coupling. This result indicates that the sensitivity of the sandwich-type immunoassay performed on the SERS substrate increases with decreasing size of sandwich immunocomplex, suggesting that the sensitivity can be improved by adopting an antibodyfragment with the same affinity for the target antigen as that of the antibody.

\section{Introduction}

Surface-enhanced Raman scattering (SERS)-based immunoassay has attracted much attention as an ultrasensitive detection method for analytes in biological samples..$^{1-4}$ The extrinsic SERS immunoassay is the platform for detecting target antigens using metal nanoparticles (MNPs), such as gold nanoparticles (AuNPs), ${ }^{5}$ gold nanostars, ${ }^{2}$ and their aggregates, ${ }^{6}$ labelled with a Raman reporter molecule and the detection antibody, which are called 'SERS probes'. ${ }^{7}$ The Raman scattering efficiency of the reporter molecule is significantly enhanced by the strong

\footnotetext{
${ }^{a}$ National Institute for Materials Science (NIMS), 1-1 Namiki, Tsukuba, Ibaraki 305-0044, Japan. E-mail: MIKI.Kazushi@nims.go.jp; Fax: +81-29-860-4718; Tel: $+81-29-860-4718$

${ }^{b}$ Faculty of Pure and Applied Sciences, University of Tsukuba, 1-1-1 Tennodai, Tsukuba, Ibaraki 305-8571, Japan

'National Nanotechnology Center (NANOTEC), National Science and Technology Development Agency (NSTDA), Pathumthani 12120, Thailand

${ }^{d}$ Department of Immunology, Faculty of Medicine Siriraj Hospital, Mahidol University, Bangkok 10700, Thailand
}

$\dagger$ Electronic supplementary information (ESI) available. See DOI: $10.1039 / \mathrm{c} 7 \mathrm{ra00154a}$ electromagnetic field near the MNP surface when the localized surface plasmon is excited by the incident laser light. Using this electromagnetic SERS effect, the target antigens can be detected with high sensitivity by measuring the Raman spectrum of the reporter molecule. Thus, the tuning of the localized surface plasmon resonance (LSPR) of MNPs, which depends on their size, shape, and material, ${ }^{8-10}$ to the excitation light is crucial for highly-sensitive detection.

The sensitivity can be improved further by performing the immunoassay on SERS-active substrates, such as two-dimensional (2D) arrays of gold nanotriangle, ${ }^{2}$ gold nanostar, ${ }^{11}$ gold nanosphere, ${ }^{12}$ and gold core-silver shell (Au@Ag) nanoparticles (NPs) ${ }^{4}$ formed on solid substrates. Many research groups reported a variety of combinations of the SERS probe and SERS-active substrate, demonstrating the ultrasensitive detection of analytes. ${ }^{3,4,13}$ This sensitivity improvement comes from the plasmon coupling between the SERS probe and the SERS-active substrate, whose strength becomes strong as the separation distance decreases. In sandwich SERS immunoassay, the capture-antibody/ antigen/detection-antibody complex (sandwich immunocomplexes) certainly acts as the separation barrier between the SERS probe and the SERS-active substrate. Thus, the size of sandwich 
immunocomplex should strongly influences the sensitivity of immunoassay. Recently, Nguyen et al. ${ }^{11}$ reported that in a sandwich SERS-based immunoassay, the SERS intensity was enhanced 2.4-fold at a certain concentration of target antigen by using Fab fragments ( $3 \mathrm{~nm}$ in size) instead of intact antibodies (13 $\mathrm{nm}$ in size). However, only a very small sensitivity improvement was observed: i.e. the slope in the sensitivity curves (the relation between the concentration of target antigen and the SERS intensity) was increased only by a factor of 1.2. Thus the influence of immunocomplex size on the sensitivity was still not confirmed.

In addition to the immunocomplex size, the metal core size of SERS probes should influence the plasmon coupling, because the strong electromagnetic field associated with the LSPR of MNPs decays with a length scale of the MNP size. ${ }^{\mathbf{1 4 - 1 6}}$ This means that the larger metal core allows larger separation distance between the SERS probe and the SERS-active substrate. Moreover, the number of Raman reporter molecules on a single SERS probe increases with increasing metal core size, resulting in the increase of Raman signal from a single SERS probe. However, for MNPs larger than $25 \mathrm{~nm}$ in size, the enhancement field at the MNP surface decreases with increasing MNP size due to the retardation effect. ${ }^{\mathbf{1 7 - 1 9}}$ Therefore, the metal core size of SERS probe must be optimized for improving the sensitivity.

To realize high-sensitive sandwich-type SERS-based immunosensors, we focus on the combined use of SERS-active substrates and SERS probes. In this study, the influence of the sizes of SERS probe and sandwich immunocomplex on the sensitivity of SERS immunoassay was investigated. Here, 2D arrays of Au (42 nm-core)@Ag (4.5 nm-shell) NPs, whose LSPR was tuned to a He-Ne laser line of $632.8 \mathrm{~nm}$, on ITO substrates ${ }^{20}$ were used as the SERS-active substrates. The SERS probes are AuNPs functionalized sequentially with 4-mercaptobenzoic acid (MBA), heterobifunctional polyethylene glycol (HS-PEG-COOH), and the antibody specific to a target antigen. MBA was selected as the Raman reporter molecule because it shows strong Raman bands at 1099 and $1596 \mathrm{~cm}^{-1}$ (ref. 21) and has a carboxyl group for antibody immobilization. First, to find the optimal SERS probe size, the sandwich immunoassay for Human-IgG (H-IgG) was performed using SERS probes of different Au core sizes from 26 to $110 \mathrm{~nm}$. The highest SERS intensity (sensitivity) was observed at an Au core size of $53 \mathrm{~nm}$. The sensitivity was improved approximately 2 -fold by using SERS probes with $53 \mathrm{~nm}$ Au core size instead of those with 26 or $110 \mathrm{~nm}$ Au core size. Next, to examine the influence of the size of sandwich immunocomplex on the sensitivity, the sandwich immunoassays for $\mathrm{H}$-IgG and prostate-specific antigen (PSA) detection were performed using the SERS probes with $53 \mathrm{~nm}$ Au core size. $\mathrm{H}$-IgG and PSA were selected as representative large and small sandwich immunocomplexes, respectively. Since the molecular weights of $\mathrm{H}$-IgG and PSA antibodies are approximately 150 $\mathrm{kDa}$, the difference in size between these two immunocomplexes comes from the size difference of antigen. The molecular weights of H-IgG and PSA antigens are approximately 150 and $30 \mathrm{kDa}$, respectively. To cancel out the difference in the immobilization ability onto the surfaces and the affinity towards target antigens between the H-IgG and PSA antibodies, the sensitivity enhancement achieved by using the SERS substrate (2D-array of Au@Ag NPs) instead of an Au evaporated film (reference substrate) was evaluated. By comparing the sensitivity enhancement factors, we found that the sensitivity of the sandwich SERS immunoassay increased by the decrease of antigen size: i.e. the decrease of sandwich immunocomplex size. This result clearly suggests that the sensitivity of SERSbased immunoassay on SERS-active substrates can be improved by reducing the size of sandwich immunocomplex, which can be achieved by replacing the antibody with an antibody-fragment with the same affinity to the target antigen. Our SERS-based immunoassay system showed a limit of detection (LOD) of $0.3 \mathrm{pg} \mathrm{mL}^{-1}$ (10 fM) for PSA and $0.05 \mathrm{pg} \mathrm{mL}^{-1}(0.3$ fM) for H-IgG.

\section{Experimental}

\section{Reagents}

Hydrogen tetrachloroaurate(III) trihydrate $\left(\mathrm{HAuCl}_{4} \cdot 3 \mathrm{H}_{2} \mathrm{O}\right)$, 1ethyl-3[3-dimethylaminopropyl] carbodiimide hydrochloride (EDC), $N$-hydroxysuccinimide (NHS), 16-mercaptohexadecanoic acid (MHDA), MBA, polyoxyethylene (20) sorbitan monolaurate (Tween 20), disodium hydrogenphosphate $\left(\mathrm{Na}_{2} \mathrm{HPO}_{4}\right)$, potassium dihydrogenphosphate $\left(\mathrm{KH}_{2} \mathrm{PO}_{4}\right)$, and sodium chloride $(\mathrm{NaCl})$ were obtained from Sigma-Aldrich. Phosphate buffered saline (PBS) solution was prepared by mixing $\mathrm{Na}_{2} \mathrm{HPO}_{4}$, $\mathrm{KH}_{2} \mathrm{PO}_{4}$, and $\mathrm{NaCl}$ : one liter of $10 \mathrm{mM}$ PBS solution includes $2.17 \mathrm{~g}$ of $\mathrm{Na}_{2} \mathrm{HPO}_{4}, 0.26 \mathrm{~g}$ of $\mathrm{KH}_{2} \mathrm{PO}_{4}, 8.71 \mathrm{~g}$ of NaCl. Silver nitrate $\left(\mathrm{AgNO}_{3}\right)$, sodium citrate, ascorbic acid, dodecanethiol, and octadecanethiol were purchased from Nacalai tesque. Polyethylene glycol 2-mercaptoethyl ether acetic acid (COOHPEG-SH, MW 3400 Da) were obtained from Nanocs, Inc. Goat anti H-IgG (\# 31119, $M_{\mathrm{w}} 150 \mathrm{kDa}$ ) was purchased from Thermo Scientific. Purified H-IgG (\# P80-105, $M_{\mathrm{w}} 150 \mathrm{kDa}$ ) was bought from Bethyl Laboratories, Inc. PSA monoclonal captureantibody (\# 10-P142A, $\left.M_{\mathrm{w}} 150 \mathrm{kDa}\right)$, PSA monoclonal detection-antibody (\# 10-7948, $M_{\mathrm{w}} 150 \mathrm{kDa}$ ), and PSA antigen (\# 30C-CP1017U, >98\% pure, $M_{\mathrm{w}} 30 \mathrm{kDa}$ ) were provided by Fitzgerald. Bovine serum albumin (BSA) was purchased from Wako. All commercial chemicals and biomolecules were used as received without further purification. Distilled water (18.2 M $\Omega$ $\mathrm{cm}$ ) was used in all experiments.

\section{SERS probe synthesis}

SERS probes with different sizes of Au cores were prepared in the following steps: (i) synthesis of citrate-capped AuNPs of different sizes, (ii) labeling and stabilizing the AuNPs with MBA and PEG molecules, respectively, and (iii) antibody immobilization. Since the detailed preparation process of the SERS probes is described in ESI, $\dagger$ here only the preparation flow is presented. Citrate-capped AuNPs of different sizes were synthesized by a seed-mediated growth method..$^{22}$ Then, the AuNPs were labeled with MBA, and subsequently treated with SH-PEG-COOH in order to acquire the dispersion stability of SERS probes. The condition of the MBA and SH-PEG-COOH treatment was optimized considering a balance between the SERS intensity and the dispersion stability of SERS probes (see 
ESI $\dagger$ ). After the solvent (DI water) of AuNPs was exchanged with a PBS solution, the carboxyl groups of MBA and $\mathrm{SH}-\mathrm{PEG}-\mathrm{COOH}$ binding to AuNPs were activated by EDC and NHS. Then, the antibody (anti H-IgG or PSA monoclonal detection antibody) was chemically immobilized on the activated AuNPs, and the non-specific binding site was blocked by BSA. After purifying the PBS solution of PEGylated MBA-labeled immunogolds, the solution was immediately used for immunoassay. When the solution could not be used immediately for some reason, it was stored at $4{ }^{\circ} \mathrm{C}$ within $10 \mathrm{~min}$, and used shortly after additional sonication for 2 s. Hereafter, the PEGylated MBA-labeled immunogolds are referred to as SERS probes, and for simplicity the size of SERS probe is specified by the Au core diameter; i.e. the $50 \mathrm{~nm}$-SERS probes are prepared from AuNPs of $50 \mathrm{~nm}$ in diameter.

\section{Sandwich immunoassay protocol}

The sandwich immunoassays for H-IgG and PSA were performed on the SERS substrates (2D arrays of Au (42 nm-core) (a) (4.5 nm-shell) NPs) and Au evaporated film substrates (reference substrates). The preparation process of both substrates was already reported in ref. 4 . The size distribution of the constituent Au@Ag NPs used in this study was $51 \pm 4 \mathrm{~nm}$ (Fig. S5 $\dagger$ ). In the last preparation step, these substrates were hydrophilized by surface modification with MHDA. The SEM image of an MHDA-hydrophilized SERS substrate was shown in Fig. S6a.† Just before immunoassay, these substrates were immersed in a mixed PBS solution of EDC and NHS (15 mM each) for $1 \mathrm{~h}$ in a dark chamber in order to activate the carboxyl group of MHDA. The unreacted reagents were removed by rinsing with PBS solution 3 times.

In the immunoassay for $\mathrm{H}$-IgG, $10 \mu \mathrm{L}$ of a PBS solution containing anti H-IgG at a concentration of $1 \mathrm{mg} \mathrm{mL}^{-1}$ was dropped onto the substrates $\left(5 \times 5 \mathrm{~mm}^{2}\right)$ and incubated overnight in a moist chamber at $4{ }^{\circ} \mathrm{C}$ for immobilizing the captureantibody. To remove the unreacted antibodies, the substrates were washed with a PBS solution containing the detergent Tween 20 at a concentration of $0.05 \%$ (TPBS) and then rinsed with PBS solution. The non-specific binding sites were blocked with BSA, which was conducted by immersing the substrates in a PBS solution with $0.05 \%(\mathrm{w} / \mathrm{v})$ BSA for $30 \mathrm{~min}$. The free BSAs were removed by sequential rinsing with TPBS and PBS solution. The immunosubstrates were stored in a PBS solution at
$4{ }^{\circ} \mathrm{C}$ until use. The SEM image of the immunosubstrate was shown in Fig. S6b. $\dagger$ Then, the antigen immobilization was performed by dropping $10 \mu \mathrm{L}$ of a PBS solution containing $\mathrm{H}$ IgG antigen onto the immunosubstrates and keeping them in a moist chamber at $4{ }^{\circ} \mathrm{C}$ for $20 \mathrm{~min}$. The unbound antigen was removed by sequential rinsing with TPBS and PBS solution. Next, $10 \mu \mathrm{L}$ of a PBS solution containing SERS probes was pipetted onto the immunosubstrates, and the solution was kept on the substrates for $10 \mathrm{~min}$ to complete the antigen-antibody binding. To remove unreacted SERS probes, the substrates were rinsed sequentially with TPBS, PBS solution, and DI water and dried under $\mathrm{N}_{2}$ gas flow. Finally, the Raman spectra of the substrates were measured.

The protocol of immunoassay for PSA was the same as that for H-IgG, except for the following conditions; the concentration of the PSA capture-antibody in PBS solution was $1 \mu \mathrm{g} \mathrm{mL}{ }^{-1}$, and the capture-antibody was incubated at room temperature (RT) for $6 \mathrm{~h}$; the incubation for immobilizing the PSA antigen was performed at RT for $3 \mathrm{~h}$; and the SERS probe immobilization was carried out at RT for $3 \mathrm{~h}$. The protocols of the sandwich SERS immunoassays for $\mathrm{H}$-IgG and PSA are illustrated in Scheme 1. The immunoassay conditions for the two systems were determined based on those reported in literatures. ${ }^{2,5,13}$ The SEM image of an immunosubstrate for PSA was shown in Fig. S6c. $\dagger$

\section{Instrumentation}

The extinction spectra of the AuNPs colloidal solutions and the SERS substrates were measured with a V-670 UV-VIS-NIR spectrophotometer (Jasco, Japan). The scanning electron microscope (SEM) images of the AuNPs, SERS substrates, and SERS probes were taken with a FE-SEM S-4800 (Hitachi, Japan). The FTIR spectra of the PEGylated-MBA-AuNPs and SERS probes were obtained with a FTIR spectrometer (Nicolet 4700) in an attenuated total reflection (ATR) geometry using a diamond prism. The Raman measurement was performed with a microRaman spectrometer system (Lambda Vision MicroRAM-300) equipped with a He-Ne laser and a charge-coupled device (CCD) detector. The excitation laser light of a wavelength of $632.8 \mathrm{~nm}$ was focused on the substrate surface with a $50 \times$ objective lens $(\mathrm{NA}=0.75)$, and the excitation power was $100 \mu \mathrm{W}$ at the surface. The laser spot size was estimated to be $4 \mu \mathrm{m}$ in diameter. The Raman scattered light was collected by the same

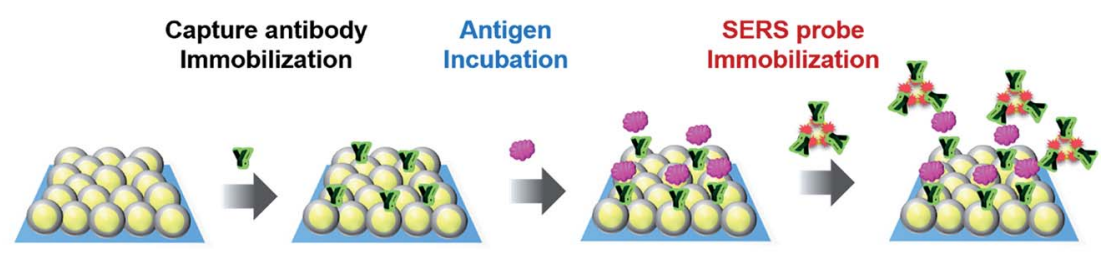

\begin{abstract}
Human IgG $10 \mu \mathrm{L}, 1 \mathrm{mg} / \mathrm{mL}$, overnight, $4^{\circ} \mathrm{C} \quad 10 \mu \mathrm{L}, 20 \mathrm{~min}, 4^{\circ} \mathrm{C}$
PSA $\quad 10 \mu \mathrm{L}, 1 \mu \mathrm{g} / \mathrm{mL}, 6 \mathrm{~h}, \mathrm{RT}$

$10 \mu \mathrm{L}, 3 \mathrm{~h}, \mathrm{RT}$
\end{abstract}

$10 \mu \mathrm{L}, 10 \mathrm{~min}, 4^{\circ} \mathrm{C}$

$10 \mu \mathrm{L}, 3 \mathrm{~h}, \mathrm{RT}$

Scheme 1 Schematic illustration of sandwich immunoassays for Human IgG and PSA. 
objective lens and dispersed with a single spectrograph with a focal length of $300 \mathrm{~nm}$ and a grating of 600 grooves per $\mathrm{mm}$. The Raman spectra were acquired with an exposure time of $50 \mathrm{~s}$. To improve the signal-to-noise ratio and reduce the data scattering originating from the spatial non-uniformity, typically the spectra measured at five different positions were averaged. The Raman measurement was performed within a day after completing the SERS probe immobilization.

\section{Result and discussion}

\section{Characterization of SERS probes}

First, we examined the size distribution and shape uniformity of the Au core particles. The SEM images of citrate-capped AuNPs of different sizes are shown in Fig. 1a, indicating that the shape is nearly sphere for all the sizes. The size distribution (average \pm standard deviation) evaluated from one hundred particles in the SEM images taken with a magnification of 100k was: $26 \pm 2 \mathrm{~nm}$, $31 \pm 2 \mathrm{~nm}, 42 \pm 4 \mathrm{~nm}, 53 \pm 5 \mathrm{~nm}, 77 \pm 8 \mathrm{~nm}$, and $110 \pm 8 \mathrm{~nm}$. The relative standard deviation was approximately less than $10 \%$ for all the sizes. The uniformity of AuNPs is crucial for examining the probe size dependence of the sensitivity of immunoassay. Fig. 1b shows the extinction spectra of the PBS solutions of SERS probes of different sizes. The maximum of the extinction band assigned to the LSPR was located at 529, 534, $543,553,575$, and $607 \mathrm{~nm}$ for the 26, 31, 42, 53, $77 \mathrm{~nm}$ and 110 $\mathrm{nm}$-SERS probes, respectively, and the band width increased with increasing particle size. Similar spectral change of the LSPR band with increasing particle size was commonly observed for aqueous colloidal solutions of AuNPs. ${ }^{18,23,24}$ The spectral change is attributed to the electromagnetic retardation effects in larger particles, which increases the contribution of higher-order plasmon modes to the extinction spectra for larger particles. ${ }^{25}$ The LSPR of the SERS probes was slightly red-shifted (2-3 nm) compared to that of the citrate-capped AuNPs of the same size, which was induced by capping AuNPs with MBA, PEG and antibody (data not shown). No broad extinction band related to agglomeration of SERS probes was observed at a longer wavelength, indicating that all the SERS probes were well dispersed in PBS solution.

Fig. 1c shows the Raman spectrum of the $53 \mathrm{~nm}$-SERS probes drop-casted on a Si substrate, together with that of solid MBA. The very strong Raman bands assigned to the $\nu_{12}$ and $\nu_{8 \mathrm{a}}$ aromatic ring vibrations ${ }^{26,27}$ were observed at 1089 and $1587 \mathrm{~cm}^{-1}$ for the SERS probes. These peak positions were slightly lower than those of solid MBA (1112 and $1607 \mathrm{~cm}^{-1}$, respectively). From these peak shifts, we confirmed that MBA was chemisorbed on AuNPs. ${ }^{26}$ In this study, the $1587 \mathrm{~cm}^{-1}$ band was used for detection of target analytes, because the $1587 \mathrm{~cm}^{-1}$ band was wellseparated from the Raman signal arising from the SERS substrates (2D-arrays of Au@Ag NPs) typically displayed at 1003, $1035,1110,1143,1180,1340,1454$, and $1530 \mathrm{~cm}^{-1}$.

The successful immobilization of antibody on the PEGylated MBA-labeled AuNPs was confirmed by measuring the FTIR spectra in an ATR geometry. Fig. 1d shows the IR spectra of the PEGylated MBA-labeled AuNPs and SERS probes drop-casted on Si substrates. In the spectrum of PEGylated MBA-labeled AuNPs, the IR bands assigned to the $\mathrm{C}=\mathrm{O}$ stretching of $\mathrm{COOH}$ group and the $\mathrm{C}-\mathrm{S}$ vibration ${ }^{28}$ were observed at 1691 and $1111 \mathrm{~cm}^{-1}$, respectively. The observation of these bands indicates the existence of MBA and/or SH-PEG-COOH molecules chemisorbed on AuNPs. After antibody immobilization, the 1691 $\mathrm{cm}^{-1}$ band disappeared and the bands assigned to the $\mathrm{C}=\mathrm{O}$ of the amide bond and the $\mathrm{N}-\mathrm{H}$ stretching of antibody appeared at (a)

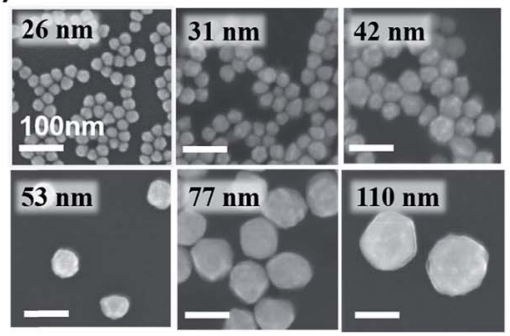

(c)

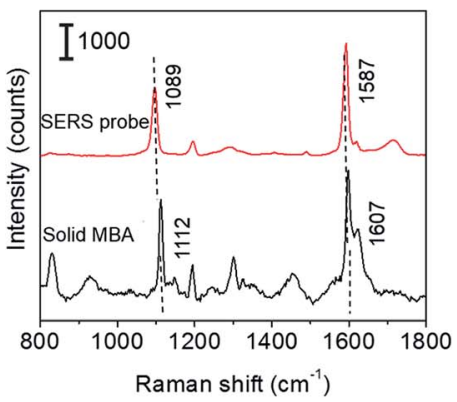

(b)

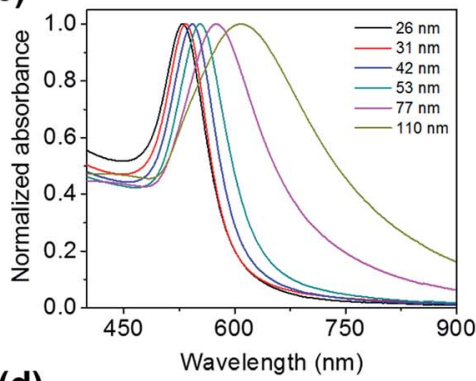

(d)

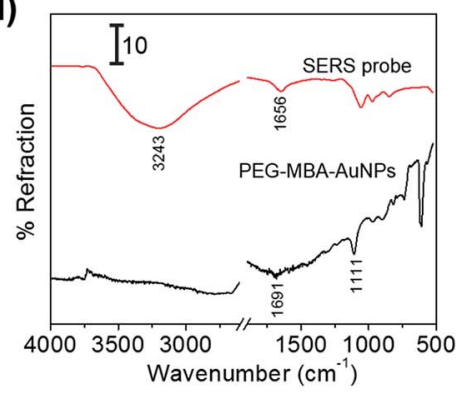

Fig. 1 (a) SEM images of the citrate-capped AuNPs of different sizes. (b) Normalized extinction spectra of the PBS solutions of SERS probes of different sizes. (c) Raman spectra of the solid MBA and the $53 \mathrm{~nm}$-SERS probes. (d) ATR-FTIR spectra of the PEGylated MBA-labeled AuNPs and the SERS probes of $53 \mathrm{~nm}$ in diameter. 
1656 and $3243 \mathrm{~cm}^{-1}$, respectively. ${ }^{29}$ From these spectral changes, the antibody was confirmed to be immobilized on the SERS probes via the amide linkage.

\section{Influence of SERS probe size on the sensitivity of SERS immunoassay}

To roughly examine the influence of the SERS probe size on the sensitivity of immunoassay, the sandwich immunoassay for $\mathrm{H}^{-}$ IgG was performed on the SERS substrates using SERS probes of different sizes $(26,31,42,53,77$, and $110 \mathrm{~nm}$ in diameter) at a constant target analyte concentration of $1 \mathrm{ng} \mathrm{mL} \mathrm{mL}^{-1}$. Fig. 2a shows the SERS spectra of the sandwich immunoassay. The intensity of the characteristic bands of MBA increased with increasing probe size until $53 \mathrm{~nm}$ and then decreased. The probe size dependence of the $1587 \mathrm{~cm}^{-1}$ band intensity is shown in Fig. 2b, which clearly shows that it becomes maximum at a probe size of $53 \mathrm{~nm}$. This result suggests that the size of the SERS probes that provide the highest sensitivity enhancement lies about $50 \mathrm{~nm}$.

The sensitivity of SERS immunoassay must be discussed based on the slope of the sensitivity curve, which is the relationship between the SERS intensity and the concentration of target analyte. Thus, we determined the sensitivity curves of the sandwich immunoassay for SERS probe sizes of 26, 53, and $110 \mathrm{~nm}$, which were selected as representative small, middle, and large probe sizes. The concentration of H-IgG antigen was varied from $0.1 \mathrm{pg} \mathrm{mL}^{-1}$ to $10 \mathrm{ng} \mathrm{mL}^{-1}$ by serial dilutions of $\mathrm{H}^{-}$ IgG antigen solution. Fig. 3 shows the sensitivity curves
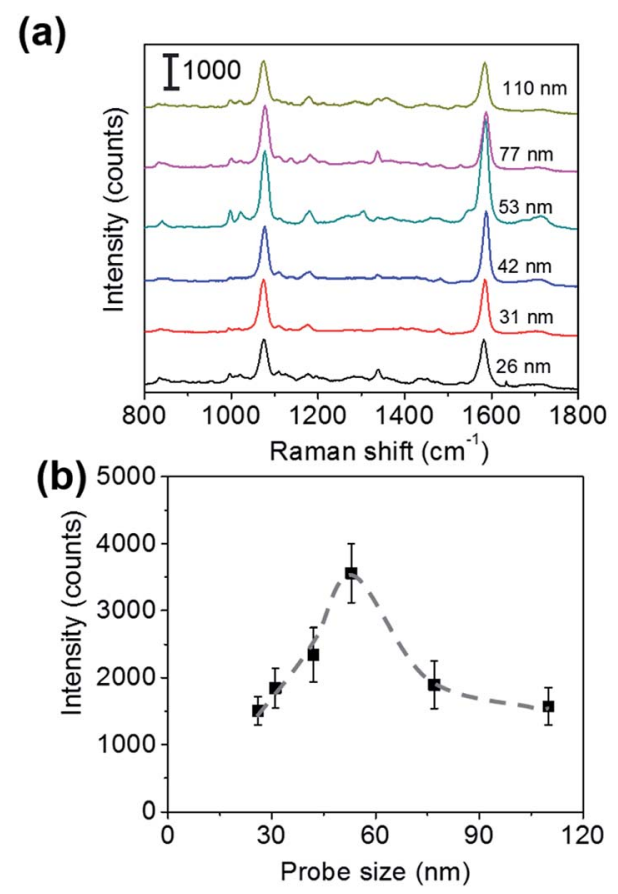

Fig. 2 (a) SERS spectra of the sandwich immunoassay for $\mathrm{H}$-IgG performed at a constant $\mathrm{H}$-lgG concentration of $1 \mathrm{ng} \mathrm{mL}^{-1}$ using SERS probes of different sizes. (b) Probe size dependence of the SERS intensity of the $1587 \mathrm{~cm}^{-1}$ band. The broken curve is a guide for the eye.

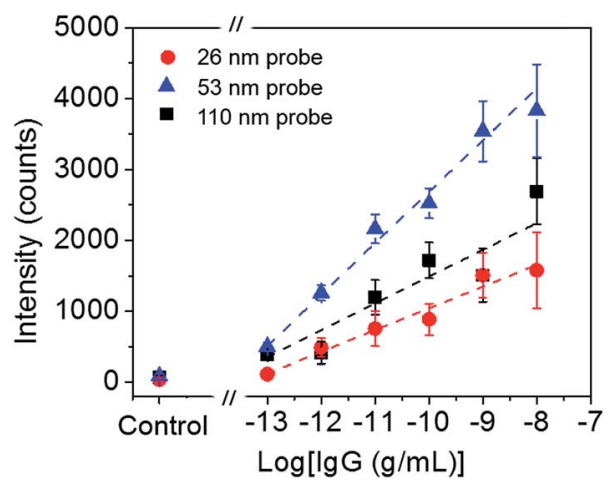

Fig. 3 Sensitivity curves of $\mathrm{H}$-lgG immunoassay using three probe size $(26,53$, and $110 \mathrm{~nm})$. The broken straight lines were obtained by the method of least squares.

obtained by plotting the $1587 \mathrm{~cm}^{-1}$ band intensity as a function of the logarithm of $\mathrm{H}-\mathrm{IgG}$ concentration. The corresponding SERS spectra are shown in Fig. S7. $\dagger$ As shown in Fig. 3, the linear relationship was obtained in the $\mathrm{H}$-IgG concentration

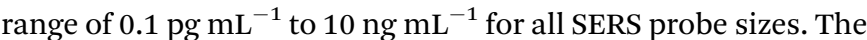
linear sensitivity curves obtained by the method of least squares are: $y=306 x+4110\left(r^{2}=0.97\right)$ for $26 \mathrm{~nm}$-probes, $y=726 x+$ $9951\left(r^{2}=0.98\right)$ for $53 \mathrm{~nm}$-probes, and $y=377 x+5268\left(r^{2}=\right.$ 0.86 ) for $110 \mathrm{~nm}$-probe. By comparing their slopes, the sensitivity of immunoassay using $53 \mathrm{~nm}$-probes was found to be approximately 2-fold higher than those using $26 \mathrm{~nm}$ - and 110 $\mathrm{nm}$-probes. This probe size dependence of the immunoassay sensitivity is in good agreement with that of the SERS intensity at a constant H-IgG concentration shown in Fig. 2b. From these results, we conclude that the optimal SERS probe size is $53 \mathrm{~nm}$. Here, we should note that the optimal size of SERS probes depends on the structure of SERS-active substrates used for immunoassay.

We would like to discuss briefly why the maximum sensitivity is observed at a SERS probe size of $53 \mathrm{~nm}$. Fig. 4a shows the representative SEM images of the immunoassay substrates performed at a constant H-IgG concentration of $1 \mathrm{ng} \mathrm{mL}^{-1}$ using SERS probes of different sizes (the corresponding SERS data are shown in Fig. 2). One can distinguish between the SERS probes and the Au@Ag NPs of the SERS substrate by the difference in size and brightness. The SERS probes situated at the top layer of 2D array appear brighter in the SEM images than the Au@Ag NPs. By counting SERS probes in the SEM images $\left(3.5 \times 2.5 \mu \mathrm{m}^{2}\right)$ taken at five different positions, their surface density was evaluated. The result is shown in Fig. 4b. The immobilized probe density decreased with increasing probe size, indicating that the immobilization probability of SERS probes decreased with increasing probe size. This may be due to insufficient binding strength of antigen-antibody for immobilizing large SERS probes.

From the observed SERS intensity $\left(I_{\mathrm{ob}}\right)$, the probe density $\left(d_{\mathrm{p}}\right)$, and the excitation laser spot diameter $\left(w_{\mathrm{L}}=4 \mu \mathrm{m}\right)$, the SERS intensity per a single SERS probe $\left(I_{\mathrm{S}}\right)$ can be calculated by $I_{\mathrm{s}}=4 I_{\mathrm{ob}} /\left(\pi w_{\mathrm{L}}^{2} d_{\mathrm{p}}\right) \cdot I_{\mathrm{s}}$ is proportional to the effective Raman cross-section of a single SERS probe. The calculated results are 
(a)

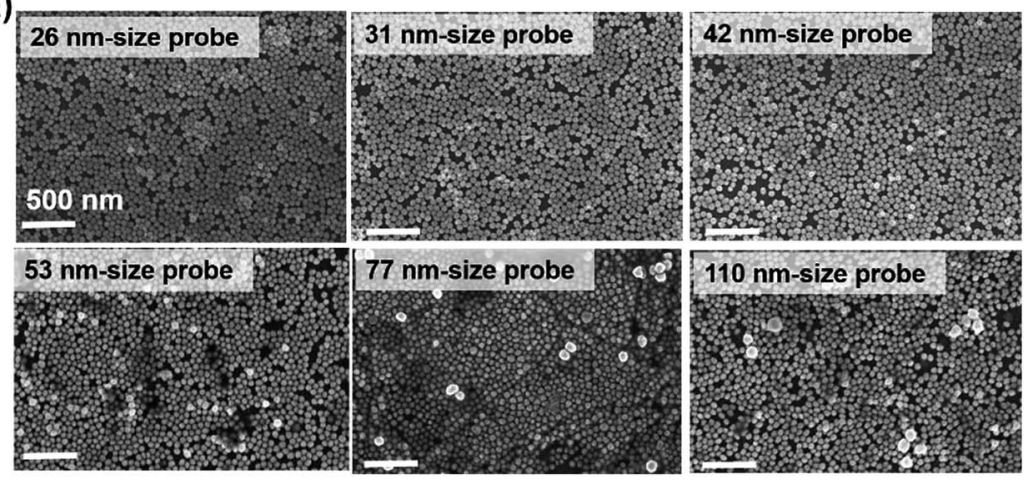

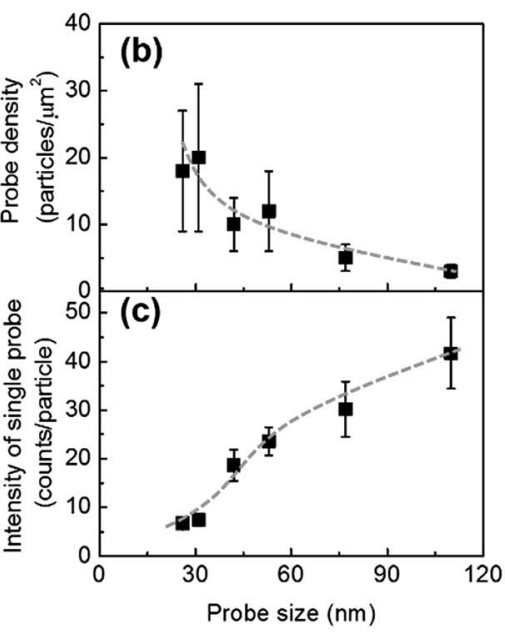

Fig. 4 (a) Representative SEM images of sandwich immunoassay substrates performed at a $\mathrm{H}$-lgG concentration of $1 \mathrm{ng} \mathrm{mL} \mathrm{m}^{-1}$ using SERS probes of different sizes. Probe size dependence of (b) probe density and (c) SERS intensity of a single probe. The broken curves are guides for the eye.

shown in Fig. 4c, indicating that the effective Raman crosssection increases with increasing probe size. This probe size dependence can be understood by considering the following two effects: one is the increase of Raman reporter molecules with increasing probe surface area, and the other is the decrease of the electromagnetic field enhancement effect with increasing probe size due to the electromagnetic retardation effect. In the probe size range of less than $110 \mathrm{~nm}$, the former effect is dominant. In consequence of the balance between the increase of $I_{\mathrm{s}}$ and the decrease of $d_{\mathrm{p}}$ with increasing probe size, the sensitivity enhancement showed a maximum around a probe size of $50 \mathrm{~nm}$.

\section{Influence of sandwich immunocomplex size on the sensitivity of SERS immunoassay}

The influence of the size of sandwich immunocomplexes on the sensitivity of SERS immunoassay was examined. PSA and H-IgG were selected as representative small and large sandwich immunocomplexes, ${ }^{30,31}$ respectively, and $53 \mathrm{~nm}$-SERS probes (optimized probe size) were used in these immunoassays. The molecular weights of the H-IgG antibody and antigen, and the PSA antibody (IgG2a isotype) were approximately $150 \mathrm{kDa}$, whose molecular size was reported to be $15 \times 10 \times 4 \mathrm{~nm}^{3} .^{32}$ The molecular weight of the PSA antigen was $30 \mathrm{kDa}$, and its molecular size was estimated to be $\sim 4 \mathrm{~nm}$ in diameter by assuming the shape of PSA antigen as a globular shape,,$^{33}$ which was in good agreement with the molecular dimension estimated by simulation. ${ }^{31}$ Thus, the size difference between the $\mathrm{H}^{-}$ IgG and PSA sandwich immunocomplexes mainly arises from the difference in size of antigen. It is very difficult to determine the size of sandwich immunocomplex that determines the separation distance between the SERS substrate and SERS probe, because it depends on the alignment structure of the capture-antibody, antigen, and detection-antibody. For simplicity, in this study the size of sandwich immunocomplex was estimated by sum of each component size. Here, the sizes of the H-IgG antibody and antigen and the PSA antibody were assumed to be $12 \mathrm{~nm},{ }^{30}$ and the size of the PSA antigen was $4 \mathrm{~nm}$.

The sensitivity curves for the detection of different analytes cannot be compared directly, because the ability of different antibodies to be immobilized onto the surface and the affinity towards their target antigens are different. Thus, the sensitivity enhancement factors obtained by replacing an Au film with the SERS substrate were evaluated for H-IgG and PSA detection, and then compared. By doing this, the influence of the size of sandwich immunocomplex on the sensitivity of SERS immunoassay could be investigated. To obtain the sensitivity curves of SERS immunoassay for H-IgG, the concentration of H-IgG antigen solution was varied from $0.1 \mathrm{pg} \mathrm{mL}^{-1}$ to $10 \mathrm{ng} \mathrm{mL}^{-1}$. For the control sample, the PBS solution without H-IgG antigen was dropped onto the substrates. Fig. S8a and $b \dagger$ show the Raman spectra of the immunoassay for H-IgG conducted on the SERS and Au film substrates, respectively. The sensitivity curves obtained by plotting the peak intensity of the $1587 \mathrm{~cm}^{-1}$ band as a function of the concentration of H-IgG are shown in Fig. 5 a. The linear relationship was observed in the concentration range of $0.1 \mathrm{pg} \mathrm{mL}^{-1}$ to $10 \mathrm{ng} \mathrm{mL}^{-1}$ for both Au film and SERS substrates. The linear relationships determined by the method of least squares are: $y=726 x+9951\left(r^{2}=0.98\right)$ for the SERS substrate and $y=310 x+4335\left(r^{2}=0.87\right)$ for the Au film substrate. By comparing the slopes, the sensitivity was found to be enhanced 2.3-fold by replacing the Au film substrate with the SERS substrate.

The sensitivity curves of the immunoassay for PSA are shown in Fig. 5b, where the concentration of PSA solution was varied

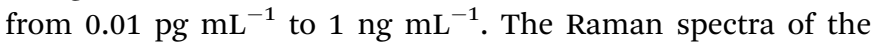
immunoassay for PSA on the SERS and Au film substrates are shown in Fig. S8c and $d, \dagger$ respectively. Fig. 5b shows the sensitivity curves of the immunoassays for PSA performed on both substrates. The linear relation was found in the range of 1 $\mathrm{pg} \mathrm{mL} \mathrm{m}^{-1}$ to $1 \mathrm{ng} \mathrm{mL} \mathrm{mL}^{-1}: y=827 x+10461\left(r^{2}=0.95\right)$ for the SERS substrate and $y=128 x+1742\left(r^{2}=0.90\right)$ for the Au film substrate. By comparing the slopes, the sensitivity of the 
(a)

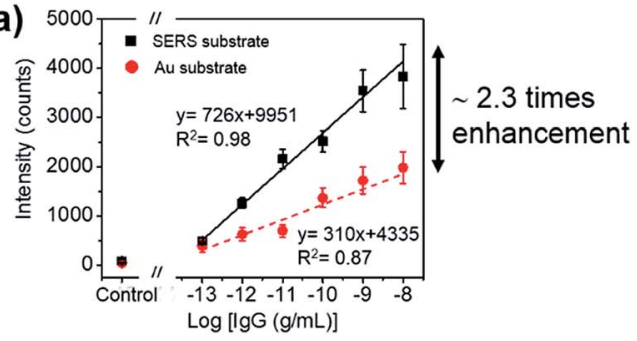

(b)

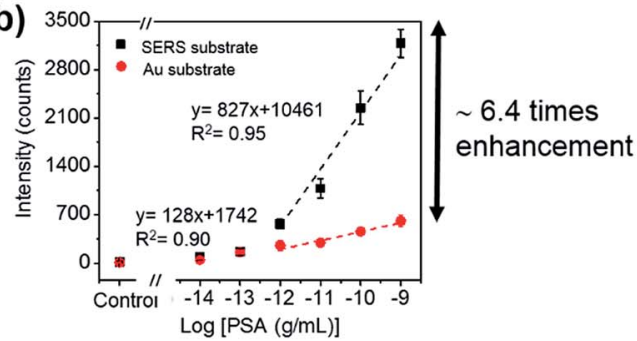

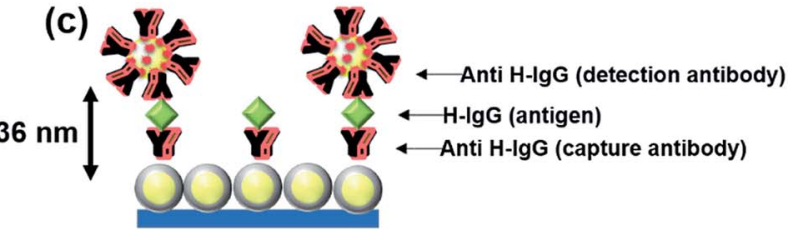

H-lgG immunoassay

(d)

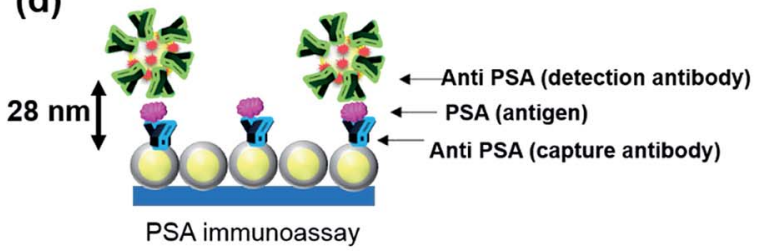

Fig. 5 Sensitivity curves of the sandwich SERS immunoassays for (a) H-lgG and (b) PSA performed on the SERS and Au film substrates. The broken lines are obtained by the method of least squares. Schematic illustration of sandwich immunoassay for (c) H-IgG and (d) PSA.

immunoassay conducted on the SERS substrate was found to be 6.4-fold higher than that of the Au film substrate.

By comparing the sensitivity enhancement factors, we found that the sensitivity of the sandwich-type SERS immunoassay increased by a factor of 3 as the size of the sandwich immunocomplex decreased from $36 \mathrm{~nm}$ to $28 \mathrm{~nm}$. This result can be understood by the increase of the plasmon coupling between the SERS probe and the SERS substrate with decreasing separation distance, suggesting that the sensitivity of SERS-based immunoassay performed on SERS-active substrates can be improved by adopting an antibody-fragment instead of the antibody. Here, we note that the reduction of the affinity to bind with the target antigen must be avoided in producing antibodyfragments.

The SERS-based immunoassay reported here showed low LODs of $0.3 \mathrm{pg} \mathrm{mL}^{-1}$ (10 fM) for PSA and $0.05 \mathrm{pg} \mathrm{mL}^{-1}(0.3 \mathrm{fM})$ for H-IgG. The LOD of our system for PSA was lower than or comparable to those of other methods reported previously: 0.1 $\mathrm{ng} \mathrm{mL}^{-1}$ for enzyme-linked immunosorbent assay (ELISA) method,,$^{34} 4 \mathrm{pg} \mathrm{mL}{ }^{-1}$ for electrochemical immunosensor using carbon nanotube-modified electrode, ${ }^{35} 10 \mathrm{fM}$ for colorimetric immunosensor, ${ }^{36}$ and $0.5 \mathrm{pg} \mathrm{mL}^{-1}$ for electrochemical immunosensor using gold nanoparticle film electrode. ${ }^{37}$ The LOD of our immunosensor for H-IgG was lower than those of fluorescent immunoassay $\left(0.8 \mathrm{ng} \mathrm{mL}^{-1}\right),{ }^{38}$ electrochemical immunoassay (1.2 $\left.\mathrm{ng} \mathrm{mL}{ }^{-1}\right),{ }^{39}$ ELISA-like assay based on gold-enhanced peroxidase-like immunogold $\left(0.3 \mathrm{ng} \mathrm{mL}^{-1}\right){ }^{40}$ and electrochemical stripping metalloimmunoassay based on silverenhanced AuNP label $\left(1 \mathrm{ng} \mathrm{mL}^{-1}\right) .{ }^{41}$

\section{Conclusion}

The influence of the sizes of SERS probe and sandwich immunocomplex on the sensitivity of sandwich-type SERS immunoassay has been investigated. Antibody-conjugated MBA-labeled AuNPs were served as the SERS probes. To enhance the SERS signal generated from the probe, the immunoassay was performed on the 2D arrays of Au@Ag NPs (SERS-active substrates). The sensitivity of immunoassay for $\mathrm{H}$ IgG detection increased with increasing probe size up to $53 \mathrm{~nm}$, and beyond this probe size it began to decrease. We found that the sensitivity could be improved by a factor of 2 by using $53 \mathrm{~nm}$ probes, instead of $26 \mathrm{~nm}$ - or $110 \mathrm{~nm}$-probes. Next, the influence of the size of sandwich immunocomplex on the sensitivity was examined by performing the sandwich immunoassay for H-IgG and PSA detection with $53 \mathrm{~nm}$-SERS probes. We found that the sensitivity of the immunoassay increased by a factor of 3 as the size of sandwich immunocomplex decreased from $36 \mathrm{~nm}$ to $28 \mathrm{~nm}$. This sensitivity enhancement can be attributed to the stronger plasmon coupling at the shorter separation distance between the SERS probe and the SERS substrate. This finding suggests that the sensitivity of sandwich SERS immunoassay performed on SERS-active substrates can be improved by replacing the capture- and detection-antibodies with antibodyfragments if the affinity of the antibody-fragments to bind with the target antigen is unchanged.

\section{Acknowledgements}

We would like to thank Dr J. Nakanishi and Dr T. Naganuma for valuable discussion. We thank Dr S. Nishiyama and Dr K. Nittoh for their technical supports. We thank both the Japan Science and Technology Agency (JST) and the National Science and Technology Development Agency (NSTDA Thailand) for financial support of the East Asia Science and Innovation Area Joint Research Program (e-ASIA JRP). A part of this work was supported by the Japan Society for the Promotion of Science (JSPS) KAKENHI Grant Number 24656040, by the Yazaki Memorial 
Foundation for Science and Technology, by NIMS and by NANOTEC. R. L., S. B., and T. D. would acknowledge funding from NANOTEC, NSTDA. K. K would acknowledge to The Ministry of Education, Culture, Sports, Science, and Technology (MEXT) for the Japanese Government Scholarship for Research Students.

\section{References}

1 J. Ni, R. J. Lipert, G. B. Dawson and M. D. Porter, Anal. Chem., 1999, 71, 4903-4908.

2 M. Li, S. K. Cushing, J. Zhang, S. Suri, R. Evans, W. P. Petros, L. F. Gibson, D. Ma, Y. Lui and N. Wu, ASC Nano, 2013, 6, 4967-4976.

3 A. Kamińska, E. Witkowska, K. Winkler, I. Dziecielewski, J. L. Weyher and J. Waluk, Biosens. Bioelectron., 2015, 66, 461-467.

4 K. Karn-orachai, K. Sakamoto, R. Laocharoensuk, S. Bamrungsap, S. Songsivilai, T. Dharakul and K. Miki, RSC Adv., 2016, 6, 97791-97799.

5 D. S. Grubisha, R. J. Lipert, H. Y. Park, J. D. Driskell and M. D. Porter, Anal. Chem., 2003, 75, 5936-5943.

6 T. Büchner, D. Drescher, H. Traub, P. Schrade, S. Bachmann, N. Jakubowski and J. Kneipp, Anal. Bioanal. Chem., 2014, 406, 7003-7014.

7 J. D. Driskell, R. J. Lipert and M. D. Porter, J. Phys. Chem. B, 2006, 110, 17444-17451.

8 T. A. El-Brolossy, T. Abdallah, M. B. Mohamed, S. Abdallah, K. Easawi, S. Negm and H. Talaat, Eur. Phys. J.: Spec. Top., 2008, 153, 361-364.

9 C. Noguez, J. Phys. Chem. C, 2007, 111, 3806-3819.

10 K. L. Kelly, E. Coronado, L. L. Zhao and G. C. Schatz, J. Phys. Chem. B, 2003, 107, 668-677.

11 A. H. Nguyen, J. Lee, H. I. Choi, H. S. Kwak and S. J. Sim, Biosens. Bioelectron., 2015, 70, 358-365.

12 K. J. Yoon, H. K. Seo, H. Hwang, D. Pyo, I. Y. Eom, J. H. Hahn and Y. M. Jung, Bull. Korean Chem. Soc., 2010, 31, 1215-1218.

13 H. Chang, H. Kang, E. Ko, B. H. Jun, H. Y. Lee, Y. S. Lee and D. H. Jeong, ACS Sens., 2016, 1, 645-649.

14 P. K. Jain, W. Huang and M. A. El-Sayed, Nano Lett., 2007, 7, 2080-2088.

15 X. Huang and M. A. El-Sayed, J. Adv. Res., 2010, 1, 13-28.

16 A. Campion and P. Kambhampati, Phys. Lett. A, 1980, 77, 381-383.

17 N. Guillot and M. Chapelle, J. Nanophotonics, 2012, 6, 064506.

18 S. Link and M. A. El-Sayed, J. Phys. Chem. B, 1999, 103, 42124217.
19 M. Kerker, D. S. Wang and H. Chew, Appl. Opt., 1980, 19, 3373-3388; Appl. Opt., 1980, 19, 4159-4174.

20 F. Pincella, Y. Song, T. Ochiai, K. Isozaki, K. Sakamoto and K. Miki, Chem. Phys. Lett., 2014, 605-606, 115-120.

21 Y. Chen, X. Zheng, G. Chen, C. He, W. Zhu, S. Feng, G. Xi, R. Chen, F. Lan and H. Zeng, Int. J. Nanomed., 2012, 7, 73-82. 22 Y. K. Park and S. Park, Chem. Mater., 2008, 20, 2388-2393.

23 S. C. Boca, C. Farcau and S. Astilean, Nucl. Instrum. Methods Phys. Res., Sect. B, 2009, 267, 406-410.

24 P. P. Fang, J. F. Li, Z. L. Yang, L. M. Li, B. Ren and Z. Q. Tian, J. Raman Spectrosc., 2008, 39, 1679-1687.

25 P. K. Jain, K. S. Lee, I. H. El-Sayed and M. A. El-Sayed, J. Phys. Chem. B, 2006, 110, 7238-7248.

26 C. Song, L. Min, N. Zhou, Y. Yang, S. Su, W. Huang and L. Wang, ACS Appl. Mater. Interfaces, 2014, 6, 21842-21850.

27 G. Varsányi, L. Láng, and M. A. Kovner, Assignments for vibrational spectra of seven hundred benzene derivatives, Adam Hilger, London, UK, 1974.

28 S. J. Patil, N. Duragkar and V. R. Pao, Sens. Actuators, B, 2014, 192, 444-451.

29 A. Barth, Biochim. Biophys. Acta, 2007, 1767, 1073-1101.

30 R. Bharadwaj, S. Mukherji and S. Mukherji, Plasmonics, 2016, 11, 753-761.

31 A. Stephenson-Brown, A. L. Acton, J. A. Preece, J. S. Fossey and P. M. Mendes, Chem. Sci., 2015, 6, 5114-5119.

32 V. R. Sarma, E. W. Silverton, D. R. Davies and W. D. Terry, J. Biol. Chem., 1971, 246, 3753-3759.

33 H. P. Erickson, Biol. Proced. Online, 2009, 11, 32-51.

34 B. Acevedo, Y. Perera, M. Ruiz, G. Rojas, J. Benítez, M. Ayala and J. Gavilondo, Clin. Chim. Acta, 2002, 317, 55-63.

35 X. Yu, B. Munge, V. Patel, G. Jensen, A. Bhride, J. D. Gong, S. N. Kim, J. Gillespie, J. S. Gutkind, F. Papadimitrakopoulos and J. F. Rusling, J. Am. Chem. Soc., 2006, 128, 11199-11205.

36 C. Cao, X. Li, J. Lee and S. J. Sim, Biosens. Bioelectron., 2009, 24, 1292-1297.

37 V. Mani, B. V. Chikkaveeraiah, V. Patel, J. S. Gutkind and J. F. Rusling, ACS Nano, 2009, 3, 585-594.

38 Z. Z. H. Fu, L. J. Hao, Y. M. Wu, H. Y. Qiao, Z. Yi, X. Y. Li and X. Chu, Anal. Sci., 2013, 29, 499-504.

39 Z. Wang, Y. Yang, J. Li, J. Gong, G. Shen and R. Yu, Talanta, 2006, 69, 686-690.

40 S. Wang, Z. Chen, J. Choo and L. Chen, Anal. Bioanal. Chem., 2016, 408, 1015-1022.

41 X. Chu, X. Fu, K. Chen, G. L. Shen and R. Q. Yu, Biosens. Bioelectron., 2005, 20, 1805-1812. 